\section{G564(P) DAILY CHANGE IN PROBABILITY OF LIVE DISCHARGE FOR EXTREME PRETERM INFANTS AT A SINGLE TERTIARY NEONATAL UNIT}

AE Bean, J Salvanos, S Clark. Jessop Wing, Sheffield Teaching Hospitals, Sheffield, UK

10.1136/archdischild-2020-rcpch.482

Aims We have experienced parents, not uncommonly, comment that 'my baby has come this far, further than we had hoped, so what is the chance of them going home now?'. This is a very challenging question to approach. This study aimed to provide a degree of objective evidence to support our communication with parents of babies born extremely preterm.

Methods Retrospective survival data was reviewed over a 7 year period at a single tertiary neonatal unit. We included all infants less than 27 weeks gestation admitted within 24 hours of birth between January 2011 and December 2017. Date of birth, gestation, birth weight, admission duration, sex, and survival to discharge data were extracted using the Badger database.

Results During the study period 403 infants born less than 27 weeks were admitted to the neonatal unit with an average gestation of 24.9 weeks, birth weight 770 grams, 56\% males and length of stay 48 days. $32 \%(n=129)$ of these babies died.

The probability of discharge alive for all infants less than 27 weeks over the 7 years was 0.6983 . This significantly rose to 0.7879 by day $4(p=0.0024)$. If an infant survived to day 13 the probability of live discharge significantly rose to $0.8425(\mathrm{p}=0.0001)$. This continued to significantly rise on day 30 to $0.9004(p=0.0367)$. The chances of survival peaked by day 84 at 0.9479 , but this was not significantly higher than at day 30 .

These figures vary by gestational age at birth and based on our study, it has been possible to create graphs for each gestation showing the probability of live discharge by day of life.

Conclusions We have shown that for extremely premature infants' the probability of life discharge at our neonatal unit is almost 0.7 and survival beyond 30 days increases the probability of discharge alive to over 0.9 .

Developing a moving probability graph of survival based on current day of life, may help families manage better with some uncertainties inherent in neonatal care. It is reassuring that parental lived experience of increasing optimism as time passes is borne out by the information in this study.

\section{G565(P) RETROSPECTIVE REVIEW OF MORTALITY IN A TERTIARY NEONATAL UNIT-IS THERE AN AUGUST EFFECT?}

AE Bean, J Salvanos, S Clark. Jessop Wing, Sheffield Teaching Hospitals, Sheffield, UK

\subsection{6/archdischild-2020-rcpch.483}

Aims Researchers have found that in adult care there is an increase in overall mortality rates in August, due to this being the time when junior doctors rotate jobs. This phenomenon has been explored by researchers in the UK and USA, with significance found by some but not others. However, this effect has not been reviewed specifically in neonatal units. Therefore, by looking specifically at survival in February and
August, we aimed to investigate any change of rotation staff effect.

Methods Badger. net neonatal health record was reviewed and data extracted for all babies admitted to the neonatal unit within 24 hours of birth between January 1st 2011 and 31st December 2017.

Simple proportions and means were calculated, with 95\% confidence intervals. Fishers Exact test was used for categorical variables. Years were compared in 3 year rolling averages as year-to-year comparisons. Infants born in the same months across the 7 years were aggregated. At the unit, the junior medical staff rotation dates are August and February. Survival rates in these two months were compared with the remainder of the year, across different gestational categories.

Results For all admissions the probability of death was highest in August $(0.075,95 \%$ CI $0.048-0.102)$ and lowest in November $(0.040,95 \%$ CI 0.019 - 0.060). However, this difference was not statistically significant. Comparison of August and February combined with all other months showed no overall significantly increased probability of death in these months for premature infants. However, when reviewing the 1558 term admissions, there was a statistically significant difference in mortality in August and February 9/233 (3.9\%) versus 21/1323 (1.6\%) for the rest of the year $(\mathrm{P}=0.0336)$.

Conclusions The significant difference in survival for term infants in August and February is an unexpected finding. It could be that staffing arrangements around junior doctor rotation may affect both antenatal and postnatal care delivery, and we suggest that more investigation into this possible effect in paediatric and neonatal care is warranted.

\section{G566(P) ACETAZOLAMIDE PARTIALLY RESTORES PULMONARY ARTERY SMOOTH MUSCLE CELL DE-DIFFERENTIATION IN EXPERIMENTAL PULMONARY HYPERTENSION}

${ }^{1,2} \mathrm{~N}$ Christodoulou, ${ }^{1,3,4} \mathrm{H}$ Hudalla, ${ }^{1,4} \mathrm{Z}$ Michael, ${ }^{1,4,5} \mathrm{H}$ Christou. ${ }^{1}$ Department of Pediatric Newborn Medicine, Brigham and Women's Hospital, Boston, USA; ${ }^{2}$ School of Medicine, Royal College of Surgeons in Ireland, Dublin, Ireland; ${ }^{3}$ Department of Neonatology, Heidelberg University Children's Hospital, Heidelberg, Germany; ${ }^{4}$ Harvard Medical School, Boston, USA; ${ }^{5}$ Division of Newborn Medicine, Boston Children's Hospital, Boston, USA

\subsection{6/archdischild-2020-rcpch.484}

Aims Pulmonary hypertension (PH) is a serious disease with limited therapeutic options. Inflammation has been shown to be causally linked to pulmonary arterial hypertension pathogenesis along with vascular smooth muscle cell (VSMC) phenotypic switching. We hypothesized that Acetazolamide (ACTZ), vis-á-vis carbonic anhydrase inhibition and induction of extracellular acidosis, restores rat pulmonary artery smooth muscle cell (RPASMC) phenotypic switching in experimental $\mathrm{PH}$.

Methods Adult male Sprague-Dawley rats were injected with Sugen $(20 \mathrm{mg} / \mathrm{kg})$ or vehicle and were exposed to hypoxia $\left(9 \% \mathrm{O}_{2}\right)$ or normoxia for 3 weeks followed by 2 days of normoxia to induce pulmonary hypertension. Lungs were harvested subsequent to induction. In vitro studies were conducted as follows: 70,000 primary RPASMCs from healthy rats were seeded in 6-well culture dishes and serum-deprived $(0.5 \%$ fetal bovine serum) for 48 hours prior to stimulation 